\title{
Design of Controller for Metal Linear Expansion Coefficient Tester Yufei FU ${ }^{1, a}$

\author{
${ }^{1}$ School of Information \& Electronic Engineering, Zhejiang Gongshang University, \\ Hangzhou 310018, China \\ aemail: fuyufei193@163.com
}

\begin{abstract}
Keywords: Linear Expansion Coefficient; Experimental Instruments; PID Algorithm; Temperature Constancy
\end{abstract}

\begin{abstract}
An intelligent metal linear expansion experimental instrument scheme brings for the existed problems aimed at measurement equipment of metal linear expansion coefficient. System by the processing core STC89C52RC adopts Holzer displacement sensor SS495A to measure displacement of metal bar expansion. Temperature sensor DS 18B20 collects the temperature of metal tube by adopting PID algorithm to control temperature as constant and using the collected data of upper computer software to obtain the final coefficient $\alpha$ of metal linear expansion. Experimental shows the scheme is steady with high accuracy.
\end{abstract}

\section{Introduction}

Linear expansion coefficient with wide application in industry is needed in the fireproofing materials to remain expansion joint for fireproofing. Linear expansion coefficient is the crucial parameter of expansion joint and masonry structure design. The appropriate linear expansion coefficient considers in the making of composite material and heterogeneous material. In addition, the measure of linear coefficient with temperature changing could analyze material mineral and propagation and coalescence of micro-crack.

There are lots of methods for measuring metal linear expansion coefficient in experimental room with limitation. For example, heating methods of vapor exist in air and water leakage, the constant temperature of vapor inlet process and the low temperature measured. The temperature of electric heating methods could not be controlled to make larger error of thermometer temperature and not to read the number of dimension telescope and thermometer at the same time for larger error in calculating data process.

This paper introduces to adopt the designing experimental instruments for metal linear expansion to measure copper linear coefficient. The coefficient $\alpha$ of metal linear expansion is obtained to collect and dispose the temperature and location data on upper computer software for processing temperature and improving accuracy.

\section{Experimental Table Construction for Metal Linear Expansion Coefficient}

The whole designing framework of hardware circuit for the design shows as figure 1 to see that the design could include keying circuit, display circuit, relay circuit, communication circuit connected on upper computer, temperature acquisition circuit adopted DS18B20 and Holzer displacement sensor SS495A acquisition circuit and power circuit. 


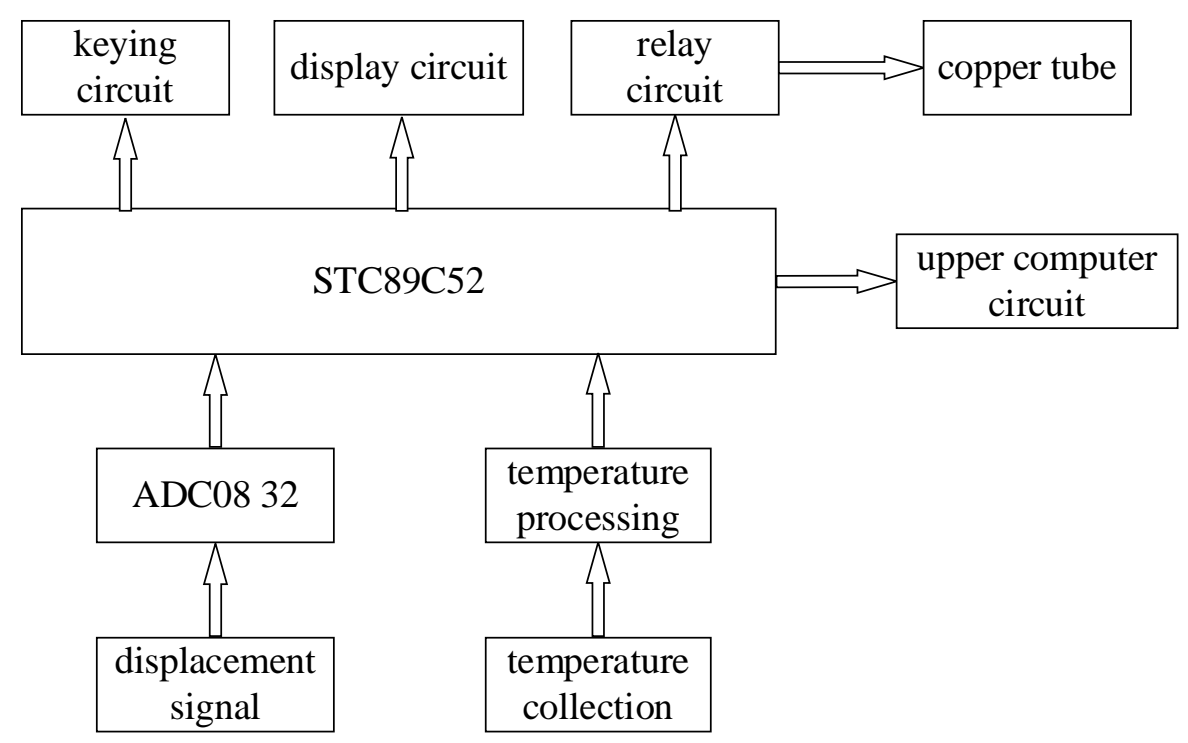

Fig.1. the whole framework for circuit

The set of experimental instrument adds the processing part with single chip core and upper computer part with VB design, specific form of experimental table for metal linear expansion coefficient shows like figure 2 including (1)metal linear expansion coefficient controller, (2) reading microscope, (3heating equipment, (4)the testing metal bar (5)upper computer. Specific construction form is for experimental table of metal linear expansion coefficient shows as figure 2.

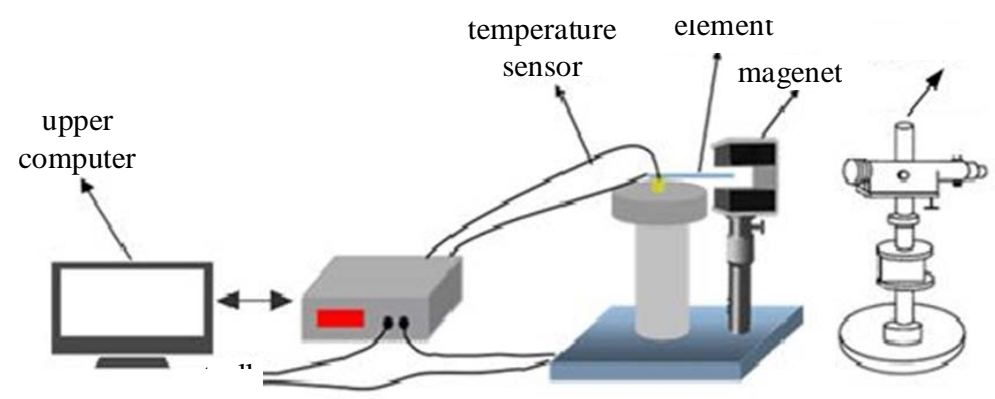

Fig.2. construction form for experimental table of metal linear expansion coefficient

\section{Software Design for Experimental Instrument of Metal Linear Expansion Coefficient}

The whole processing principle structure shows as figure 3, data setting input end sets the aimed temperature value disposed by single chip data processing center, drive relay heats metal tube and PID algorithm goes on for the setting temperature value and actual temperature value, single chip emits pulse signal close to expectation value to make temperature of metal tube same with the expectation value. Temperature procession is in design to collect voltage changing of displacement sensor result from metal tube location changing with communication for upper computer.

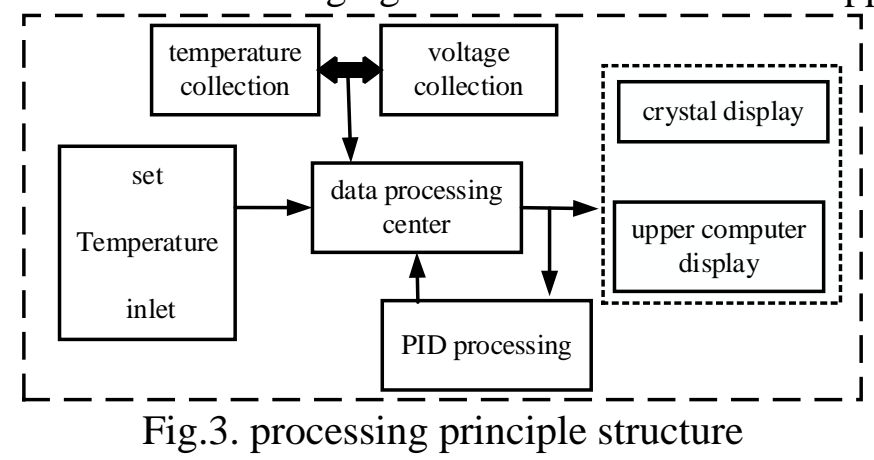

\section{Software design for experimental instrument controller of metal linear expansion coefficient}

Software design includes main programming design, regulation programming design of 
temperature PID, communication programming design of single chip and upper computer, keying testing programming design, programming design related with 1602 liquid crystal, reading programming design of temperature and voltage. The following parts introduce the main programming flow chart, programming flow chart of temperature PID regulation, like figure 4 and 5 .

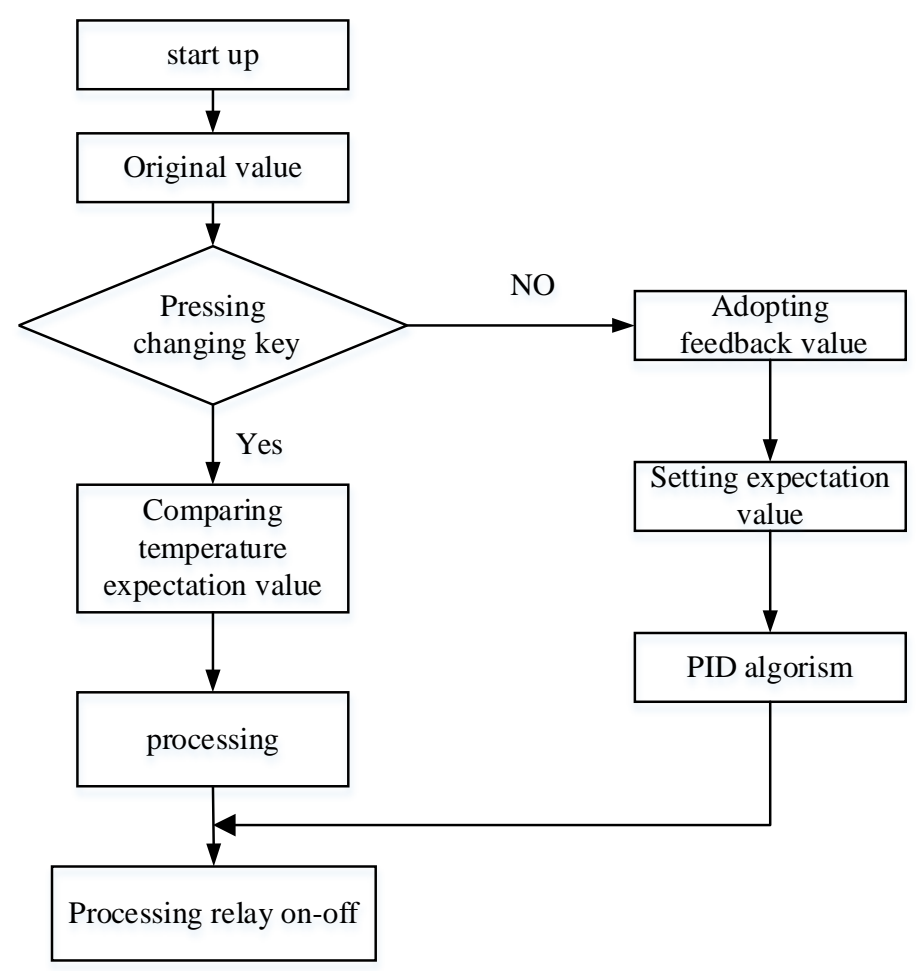

Fig.4. main programming flow chart 


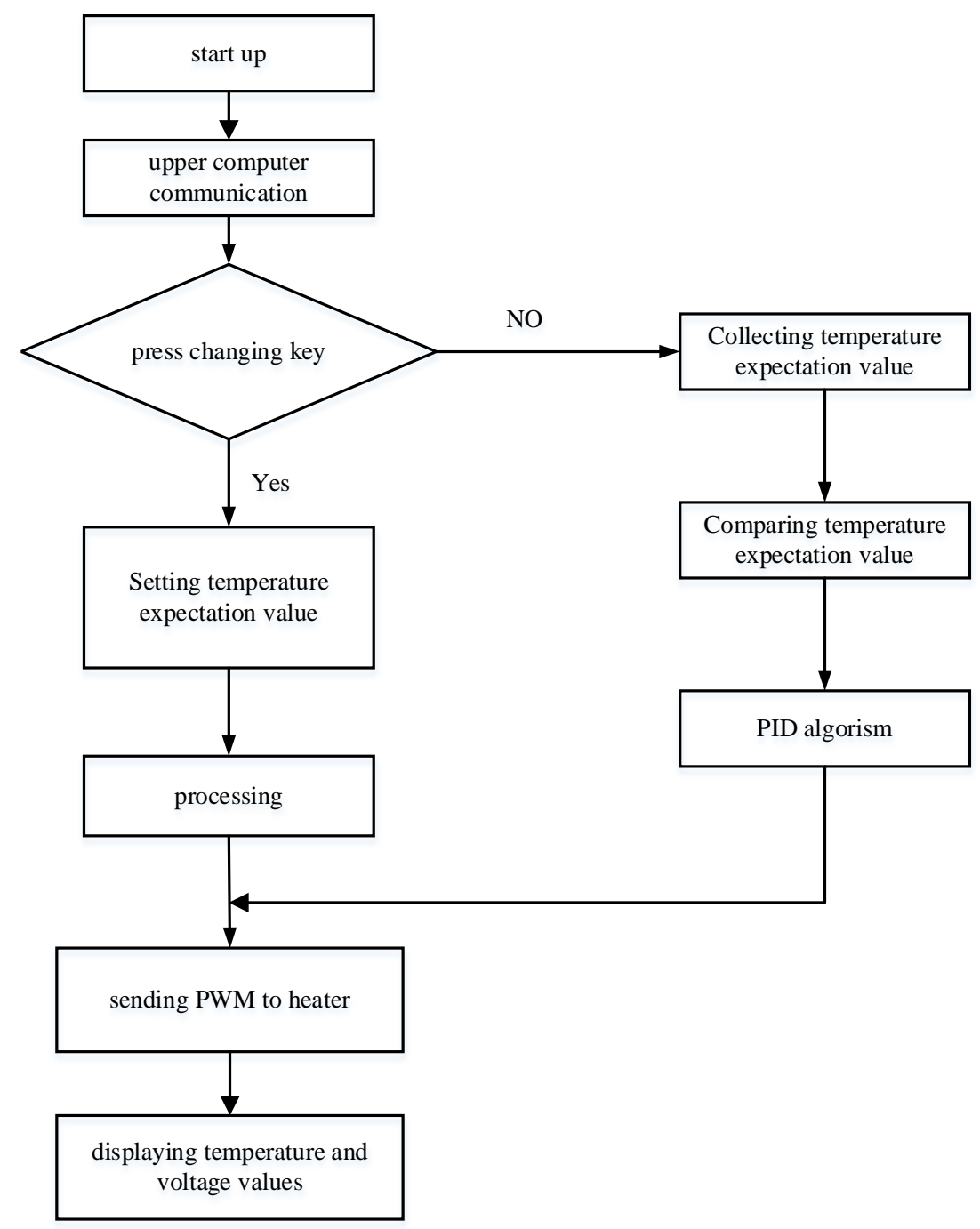

Fig.5. programming flow chart of temperature PID regulation

\section{Software design for VB upper computer}

Writing upper computer software makes single chip send data to computer by communication of serial port and upper computer for displaying on VB interface. The data sent draws the curve of voltage and temperature to get metal linear expansion coefficient $\alpha$. The operational interface is shown like figure 6 .

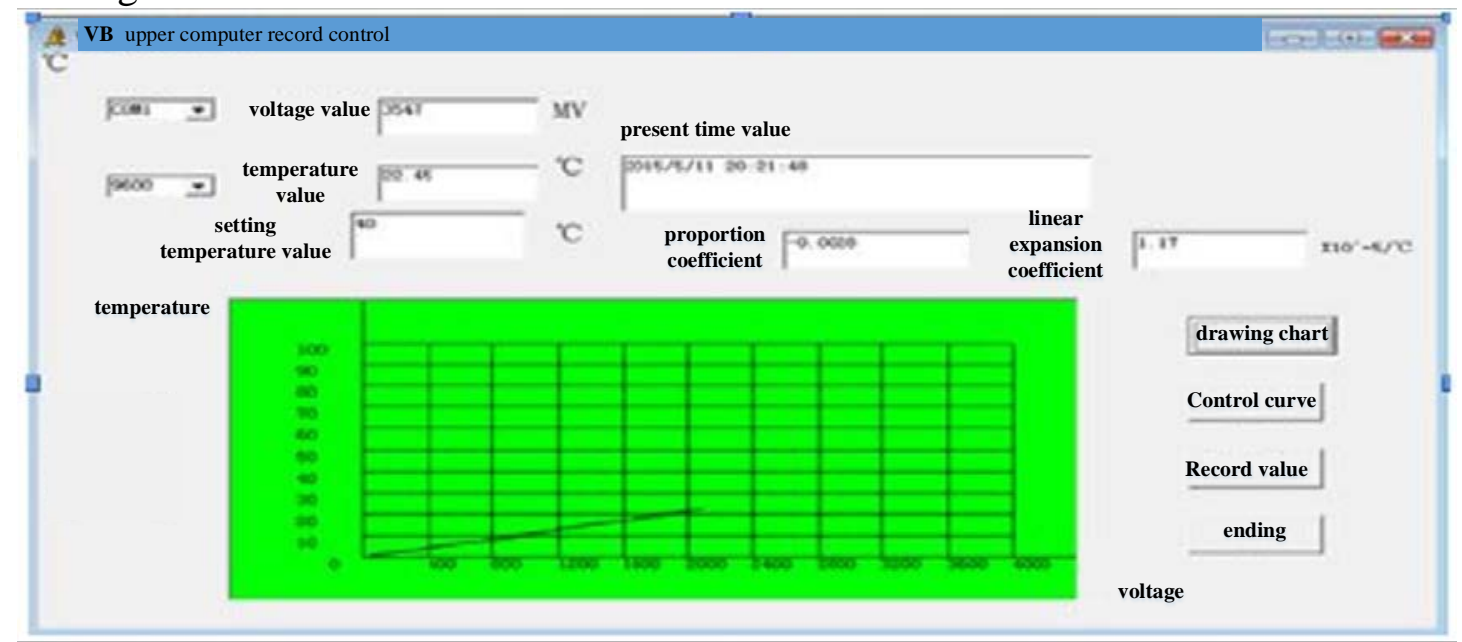

Fig.6. VB data acquisition interface

The interface shows the linear relation of voltage and temperature. The voltage and temperature on interface could be on real-time display and the testing data could be stored in the corresponding content to show for classmates. 


\section{Debugging and Experiment}

\section{Debugging for PID parameter}

The PID processing rules could be shown as the following formula:

$G_{C}(S)=K_{p}+\frac{K_{1}}{S}+K_{D} S$ consists in three parts like proportion (P), integration (I) and differential (D). The structure framework figure is as shown 7.

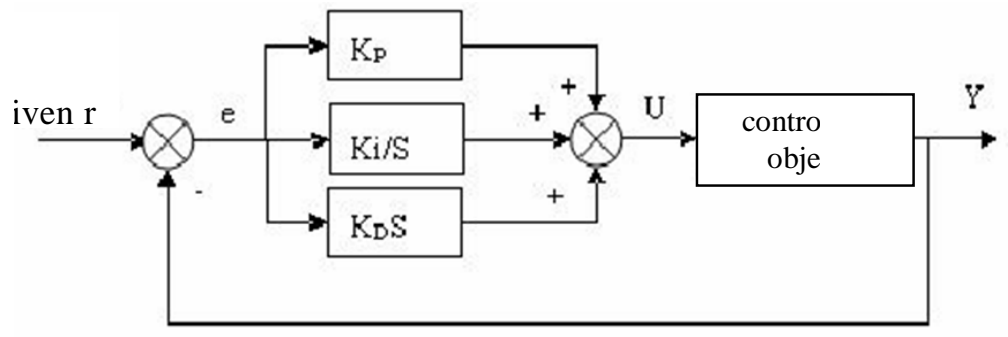

Fig.7. PID structure chart

The gathering and trying methods could be confirmed the three parameter of PID. But the general characteristic for three parameter of P, I, D should be known at first: (1)When proportionality coefficient $\mathrm{P}$ adds, response speed of device could be fastened, but the overshoot of system enlarges more further to decrease stability. (2) Variable of integration I enlarges to restrain the overshoot of device to make device steady, but the time of eliminating device error improves. (3) Derivative time adds to make device response speed fast and device overshoot decrease, but device takes easily interference outside.

The experiment adopts copper tube as testing object, copper tube heating system has two stage transfer function by looking for related information and the transfer function model for heating system of copper tube is:
(1) $G_{s 1}=\frac{9.88}{120 s+1} e^{-80 s}$,
(2) $G_{s 2}=\frac{0.105}{10 s+1}$

Establishing SIMULINK model is like figure 8 by MATLAB software to find and set $k p$ 、 $k i$ and $k d$ of system. When $k p=1.551 ; k i=0.00857 ; k d=50.59$, simulation is on for copper cube heating temperature. It could be found from curve of figure

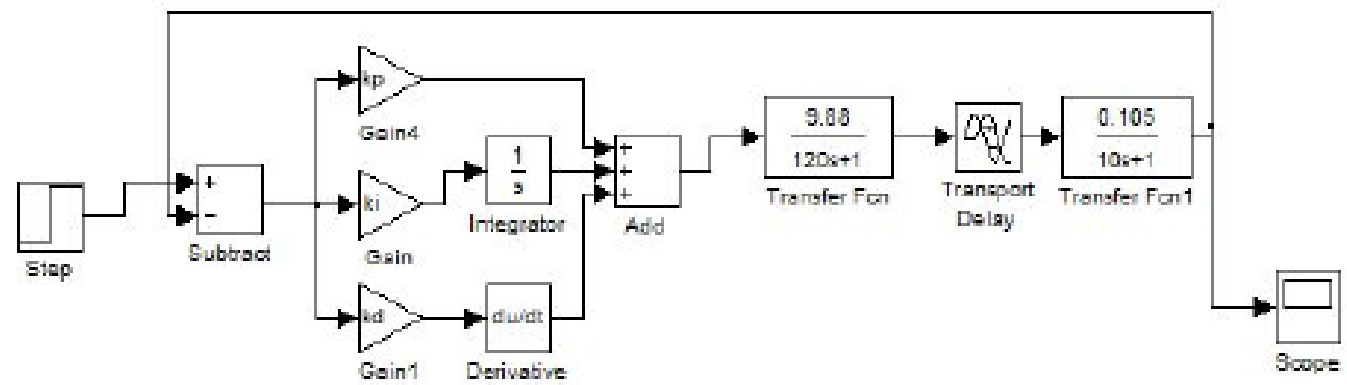

Fig.8. simulation chart for SIMULINK model of copper cube heating

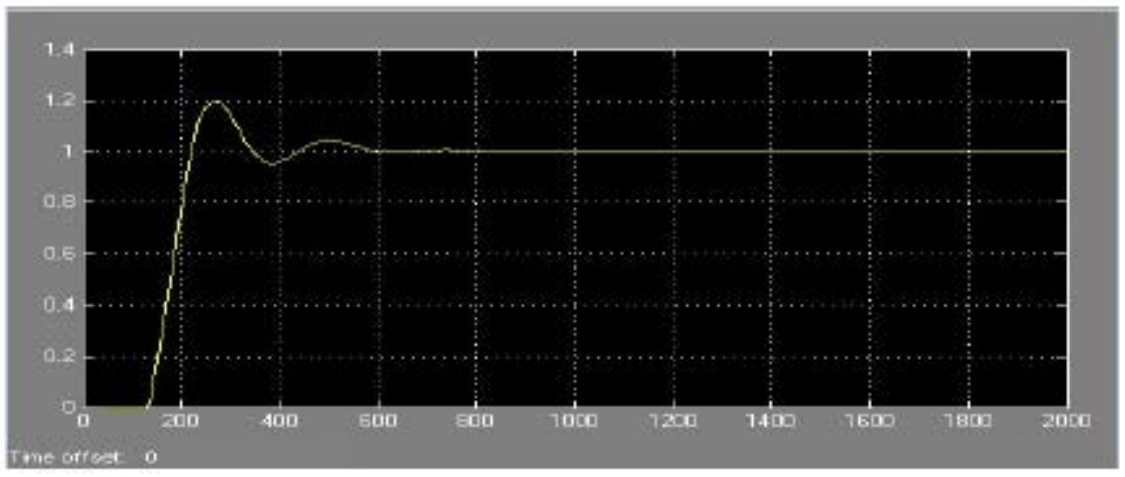

Fig.9. PID simulation chart for copper tube heating 


\section{Experiment}

(1) Experimental principle

When metal temperature rises, metal tube heating adds the metal tube length. The length $L$ of metal tube and temperature $\theta$ generally could have the following relation:

$L=L_{0}(1+\alpha \theta)$

$L_{0}$ is the length of solid at $\theta=0, \alpha$ is linear expansion coefficient. The value is related with material property, unit is ${ }^{\circ} \mathrm{C}^{-1}$. When temperature rises, the length of tube is $L$ at $\theta_{1}{ }^{\circ} \mathrm{C}$. When temperature rises by $\theta_{2}{ }^{\circ} \mathrm{C}$, the length adds $\Delta L$. According to formula (1), it concludes that:

$$
\alpha=\frac{\Delta L}{L\left(\theta_{2}-\theta_{1}\right)-\Delta L \theta_{1}}
$$

Due to $\Delta L=L,(2)$ could be written as :

$$
\alpha=\frac{\Delta L}{L\left(\theta_{2}-\theta_{1}\right)}
$$

(2) Experimental methods

The copper tube should be ready for experiment, experimental table constructs as figure 1 . Opening controller is at first and scaling is for Holzer displacement sensor SS495A. Making notes is for voltage and temperature. Temperature sensor has larger measurement range and better accuracy than the former mercurial thermometer, the great rising is for data accuracy.

(3) Measurement results and discussion

Firstly, SS495A Holzer element is scaling, and then Holzer element is scaling by reading microscope. The experimental data is shown like figure 1.

Tab.1. scaling experimental data for reading microscope of Holzer element

\begin{tabular}{cc} 
Voltage $(\mathrm{mv})$ & Location $(\mathrm{mm})$ \\
\hline 2882 & 1.244 \\
2867 & 1.195 \\
2850 & 1.150 \\
2838 & 1.085 \\
2821 & 1.032 \\
2804 & 0.974 \\
2793 & 0.925 \\
2784 & 0.892 \\
2768 & 0.838 \\
2751 & 0.774 \\
\hline
\end{tabular}

Adopting origin proceeds fitting curve for location reading and Holzer voltage during dealing with data, figure 10 is linear fitting chart of location $L$ and voltage. The curvilinear equation after dealing is: 


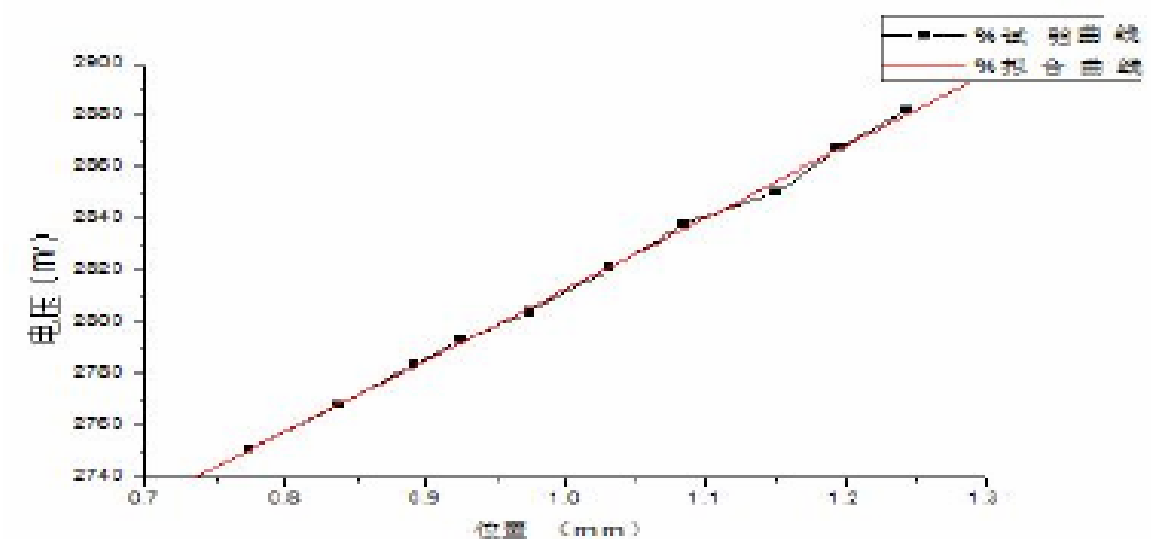

Fig.10. linear fitting chart of location $L$ and voltage

Tab.2. metrical data of temperature, voltage and displacement

\begin{tabular}{ccc}
\hline Temperature $/{ }^{\circ} \mathrm{C}$ & Holzer sensor voltage $(\mathrm{mv})$ & Displacement $(\mathrm{mm})$ \\
\hline 20.34 & 2882 & 1.244 \\
28.75 & 2862 & 1.177 \\
38.91 & 2838 & 1.085 \\
45.83 & 2821 & 1.032 \\
52.98 & 2804 & 0.974 \\
61.47 & 2784 & 0.892 \\
67.84 & 2768 & 0.838 \\
76.09 & 2748 & 0.764 \\
84.34 & 2729 & 0.695 \\
93.01 & 2708 & 0.620 \\
100.08 & 2692 & 0.559 \\
107.85 & 2673 & 0.492 \\
115.44 & 2656 & 0.429 \\
123.78 & 2637 & 0.361 \\
131.03 & 2619 & 0.295 \\
140.26 & 2597 & 0.215 \\
\hline
\end{tabular}

The location display and temperature could be obtained by using origin software: $y=1.422-0.00861 * x$, so it would know $\alpha=\frac{1}{L} \times k=17.2 \times 10^{-6}{ }^{\circ} \mathrm{C}^{-1}$, analyzing relative error: $E_{\alpha}=\frac{\left|\alpha_{0}-\alpha\right|}{\alpha_{0}} \times 100 \%=0.2 \%$, the measurement results are accurate. (standard value of copper tube $\alpha_{0}=17.1 \times 10^{-6}{ }^{\circ} \mathrm{C}^{-1}$ )

\section{Conclusion}

This paper designs an intelligent experimental instrument of metal linear expansion, system makes STC89C52RC as processing core, the number intelligence acquisition is for temperature and displacement by sensor, PID algorithm makes metal tube temperature constant, the acquisition data disposes by upper computer software, at final, the metal linear expansion coefficient a obtains directly. The designing scheme is operated on stage: experimental instrument is steady with higher accuracy data. 


\section{References}

[1] Shuang Zhou, Liang Mi, Hao Chen, Yishuang Geng, Building detection in Digital surface model, 2013 IEEE International Conference on Imaging Systems and Techniques (IST), Oct. 2012.

[2] Jie He, Yishuang Geng, Kaveh Pahlavan, Toward Accurate Human Tracking: Modeling Time-of-Arrival for Wireless Wearable Sensors in Multipath Environment, IEEE Sensor Journal, 14(11), 3996-4006, Nov. 2014.

[3] Lv Z, Halawani A, Fen S, et al. Touch-less Interactive Augmented Reality Game on Vision Based Wearable Device[J]. Personal and Ubiquitous Computing, 2015, 19(3): 551-567.

[4] Guanqun Bao, Liang Mi, Yishuang Geng, Mingda Zhou, Kaveh Pahlavan, A video-based speed estimation technique for localizing the wireless capsule endoscope inside gastrointestinal tract, 2014 36th Annual International Conference of the IEEE Engineering in Medicine and Biology Society (EMBC), Aug. 2014. 